\title{
Creating Industrial Network with PROFINET Communication for Education Purposes
}

\author{
Martin Višňovský ${ }^{1, *}$, Róbert Rákay ${ }^{1}$, Alena Galajdová ${ }^{1}$, Dušan Šimšík ${ }^{1}$ \\ ${ }^{1}$ Technical University of Košice, Faculty of Mechanical Engineering, Institute of Automation, Mechatronics and Robotics, Department of Automation, \\ Control and Human-Machine Interaction, Košice
}

\begin{abstract}
This article describes the creating of industrial network between multiple PLCs and also their mutual communication via PROFINET. Currently, industrial networks with PROFINET communication are used in industrial practice very often and therefore is necessary to teach students in automation specialization how to build such networks and how those networks work.
\end{abstract}

Keywords: Industrial network, PROFINET, PLC (Programmable Logic Controller), HMI (Human Machine Interface).

\section{Introduction}

At present, the management of industrial processes, production lines and machines make extensive use of programmable logic controllers (PLC). Thanks to them, the process can be divided into smaller sections to create a distributed control system.

One PLC could not create and manage the whole system. Controlling devices on higher-level are needed, but especially important is communication between the PLCs and the higher-level control system via an industrial network.

Industrial networks today are most often formed by twisted pair cable connections. Via this communication channel is realized an industrial communication using of various protocols. The particular protocol is usually given by manufacturer of a equipment used in the industrial network.

This article describes a simple demonstration of creating industrial network, consisting of two PLC and two HMI panels. Their connection is realized via the twisted pair cables.

The objective of creating this small industrial network is to describe the principles of creating such network, and demonstration of the principles and functions of the communication that takes place in that network. This sample is used in the learning process for students of automation at our Faculty.

Students of field automation during the study should get not only theoretical knowledge, but they should be able to solve practical problems. Therefore, the individual subjects proposed and created practical problems in order to verify the theory in practice. For these laboratory tasks, we use the most advanced technology from companies operating in industrial automation.

\section{Used devices and softwares}

Devices and softwares, which were used in creation of presented industrial network are from SIEMENS. Used devices were PLCs, HMI panels and switches. Used softwares were TIA Portal and Proneta. 


\subsection{PLC}

A simple industrial network has been created using two PLCS SIEMENS Simatic S7-1200 with the compact CPU 1214C AC/DC/Rly (Figure 1). The basic rack contains fourteen digital inputs 24V DC, ten digital outputs implemented as relays 2A, two analog inputs with a range of 0-10V DC. It has an integrated power supply for 85 - 264V AC with a frequency of $47-63 \mathrm{~Hz}$. Memory size for program and data is $100 \mathrm{kB}$. This PLC has one network card with RJ-45 connector for PROFINET communication. PLC is via this port physically connected to industrial network. [1]

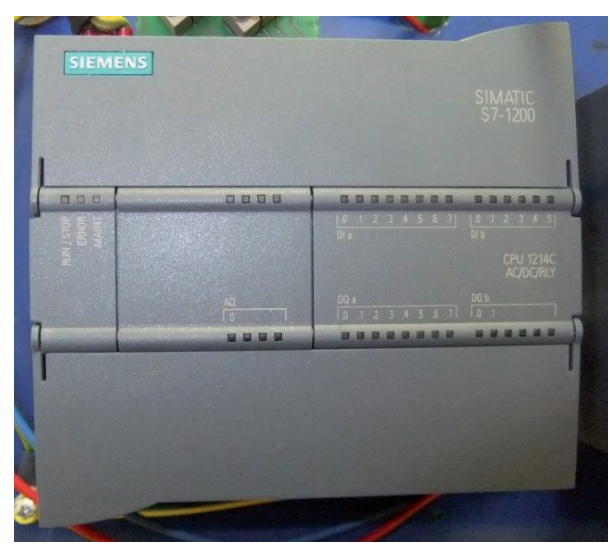

Fig. 1: Simatic S7-1200 CPU1214C AC/DC/RLY.

\section{$2.2 \mathrm{HMI}$}

Operator panels for human-machine interactions - HMI panels are nowadays frequently used devices in automation. Their main functions are visualization, monitoring and control of processes that are currently running. Operator can intervene to the process via HMI panel. In our case, we used HMI panel SIEMENS KTP700 Basic PN (Figure 2). It is seven inches touch widescreen with colour display and eight function keys. It's a basic HMI panel for easy and medium difficulty tasks, designed for connection to PLC SIEMENS from series S71200. The panel includes USB and RJ-45 ports,for connection to our network. [2].

\subsection{SWITCH}

SCALANCE $X$ is the product group of industrial switches from SIMATIC NET for Industrial Ethernet. Switches are active network components that specifically distribute data to the relevant addressees. The switch SIEMENS SCALANCE XB005 (Figure 3) is the last device that was used in our model. It's a simple unmanaged Industrial Ethernet

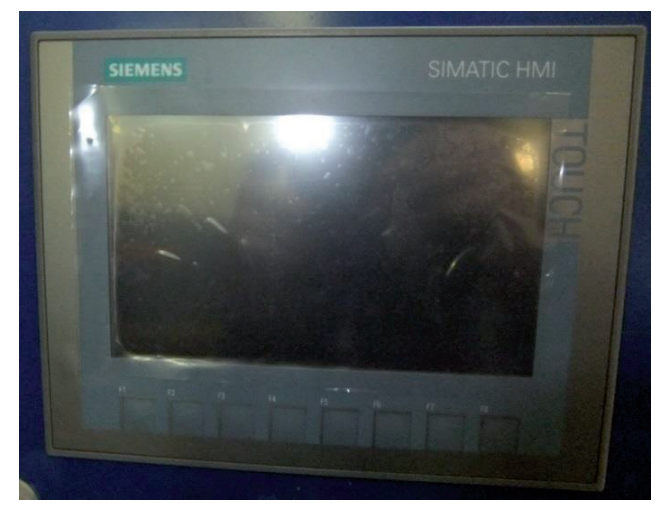

Fig. 2: SIEMENS KTP700 Basic PN

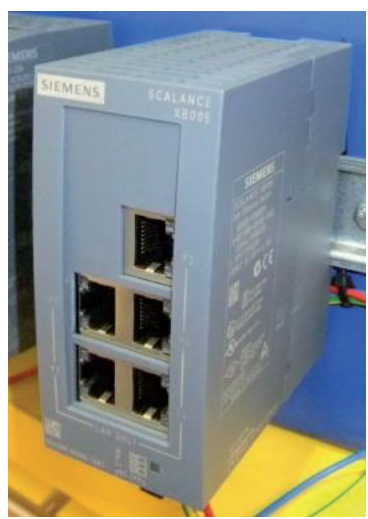

Fig. 3: SIEMENS SCALANCE XB005

switch with supported transfer rates 10/100Mbit/s. It includes five RJ-45 ports that support these transmission speeds. The functionality of each port can be controlled via the LEDs on each port. This switch is suitable for use in small networks with line or star topology. It is powered by $24 \mathrm{~V} \mathrm{DC}$. [3].

\subsection{TIA PORTAL}

TIA Portal V13 SP1 version Basic is software we used for the network configuration (Figure 4). It is a compact engineering software that minimizes time of configuration. TIA Portal joins two, previously separated softwares. The first software, which is integrated into the TIA Portal is STEP 7 Professional. This software in Basic version of TIA Portal is used for programming PLCs Simatic S7-1200 series and also allows to configure these PLC with Simatic HMI Basic panels. The second software is the WinCC Basic, which is used to program the HMI panels.

SIMATIC STEP 7 Basic offers the same benefits as STEP 7 Professional engineering software thanks to its integration into the TIA Portal engineering framework, e.g. direct online diagnostics, easy 
creation of technology objects and also the library concept for time-saving, efficient work and programming components reuse.

SIMATIC STEP 7 Basic supports IEC programming languages such as LAD (Ladder Diagram), FBD (Function Block Diagrams) and SCL (Structured Text). [4]

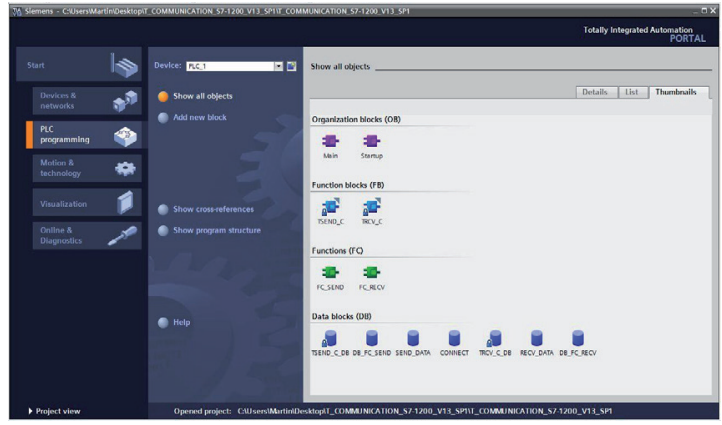

Fig. 4: TIA Portal V13 SP1Basic - workspace.

\subsection{PRONETA}

Proneta software (Figure 5) is a SIEMENS product. It simplifies the configuration of the PROFINET network and its commissioning. Network topology is automatically detected. Parameters of IP addresses for individual PROFINET device can be adjusted manually or can be easily used parameters of templates that can be created by Proneta.

Thanks to the program Proneta, we can simply configure, control and monitor $1 / O$ modules SIMATIC ET 200SP, ET 200MP, ET 200AL, ET 200eco PN and ET 200S distributing I/O systems. The test results are easy to view on the score report.

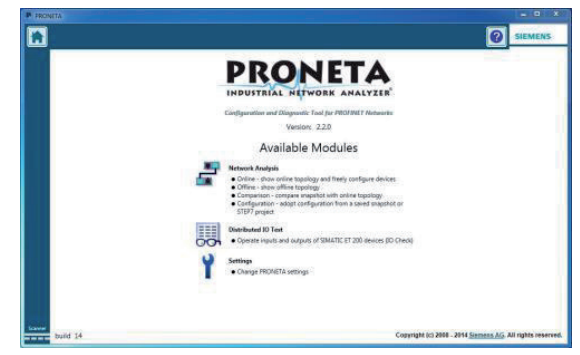

Fig. 5: Proneta.

\section{Hardware configuration}

We used TIA Portal for hardware configuration. At first, we inserted two PLCs Simatic S7-1200 CPU1214C AC/DC/RLY with product code 6ES7 214-1BG40-0XB0. Then, we assigned to those PLCs two HMI panels KTP700 Basic PN with product code
6AV2 123-2GB03-0AX0. Finally in that program we connected those devices into one PROFINET network with the label PN/I_1 (Figure 6).

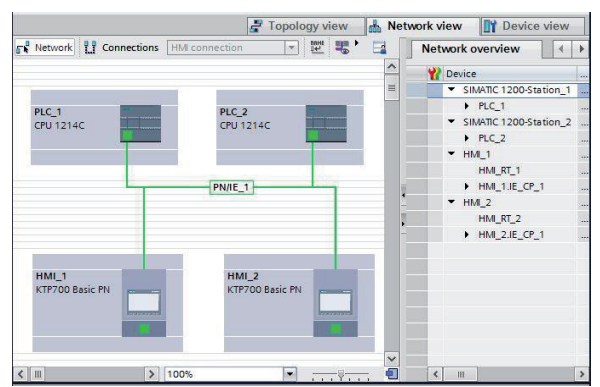

Fig. 6: Hardware configuration with network connection.

This connection we realized with twisted pair cables with connectors RJ-45. Used devices have only one RJ-45 port, therefore physical connection is implemented via two switches Scalance XB005.

One PLC and one HMI are connected to each of two switches, and both switches are connected together. This connection could be implemented even with only one switch, but because stands physical design and the possible extension of the network, we used two switches (Figure 7). Into one of the available ports on the switch we connected PC, from which we configured hardware in our network, wrote and recorded programs for individual devices.

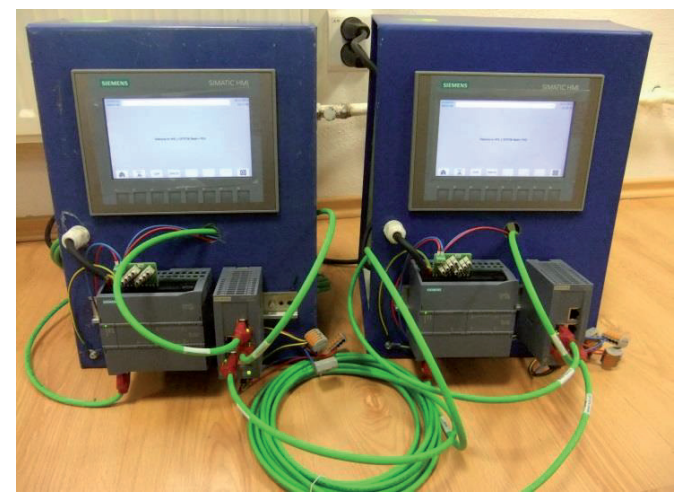

Fig. 7: Physical connection of devices.

\section{Network configuration}

Proneta software was used for PROFINET network configuration. After connecting a PC to the PROFINET network and start the software, Proneta automatically finds available devices in the network. To these devices we can assign the required IP address and subnet mask. We must to 
do correct configuration of the network card in the PC. All devices must be in the same subnet to enable their mutual communication. List of set values is in Table 1.

Tab. 1: List of devices and their network parameters.

\begin{tabular}{|l|l|l|}
\hline Device & \multicolumn{1}{l|}{ IP address } & \multicolumn{1}{l|}{ Subnet mask } \\
\hline PLC 1 & 192.168 .0 .1 & 255.255 .255 .0 \\
\hline HMI 1 & 192.168 .0 .2 & 255.255 .255 .0 \\
\hline PLC 2 & 192.168 .0 .3 & 255.255 .255 .0 \\
\hline HMI 2 & 192.168 .0 .4 & 255.255 .255 .0 \\
\hline PC & 192.168 .0 .10 & 255.255 .255 .0 \\
\hline
\end{tabular}

After correct configuration, we tested functions of connection via Proneta. Flashing LEDs on individual devices and green color of devices blocks in Proneta signalize correct communication (Figure 8).
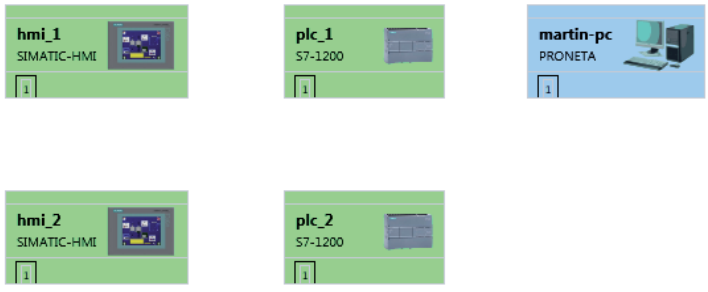

Fig. 8: Network in Proneta.

Physical connections between devices on the previous figure of network are not displayed. The absence of the lines was caused because Proneta cannot detect connections via unmanaged switch and this type of device.

\section{Program for PLC}

PLC programming was realized via TIA Portal. The goal was to create a program, which allows duplex communication between two PLC. The program consists of more blocks. There are different types of these blocks.

The first type of blocks are OB (Organization Bocks). In the program there are two blocks of this type. The first is the OB1, as standard in every Simatic program. In this case we assigned to it name Main. This block is performed cyclically around. It contains calls of functions for sending data (FC_ SEND) and data receiving (FC_RECV). It also contains commands for registration of states of digital inputs to data block, which will be sent and commands for registration of received values from data block to PLC memory. The second block is OB100. This block is called Startup. In the body of this block is only one command to set value of memory variable M0.1 to 1. This variable will be used in the program to turn on and off the communication.

The other type of blocks are FC. In the program two are used. One for sending data and the other for receiving data. These two functions are the main core of the whole program. Thanks to them is the communication allowed between the PLC.

Function for sending data is FC_SEND. It's base is function block (FB) called TSEND_C (Figure 9). [5]

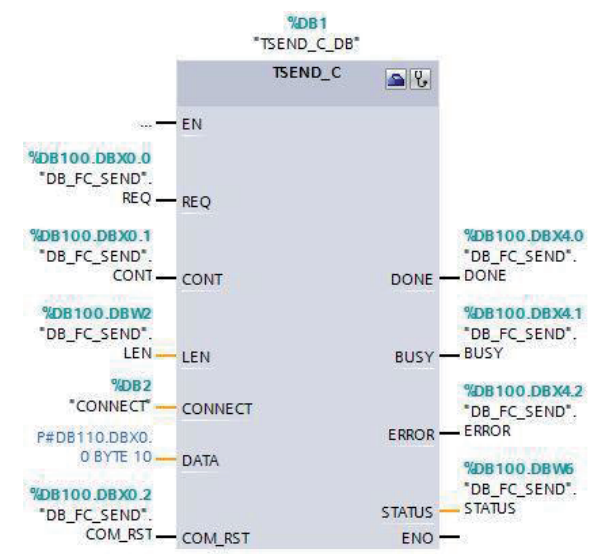

Fig. 9: Function block TSEND_C with inputs and outputs.

Every inputs of this function block are registered in data block DB100. The first input REQ is BOOL type and launches the whole process of sending on rising edge of signal 1. The second input is CONT. It's BOOL type and controls the communication. For value 0 disconnects communication connection, for value 1 establishes and maintains the communication connection. The third input is LEN. This input is UINT and defines the maximal count of bytes, which can be sent. Fourth input is CONNECT. It is a pointer, which is linking to a data block, where are described the details of communication connection. Fifth input is DATA. It's pointer, which is linking to data block, in our case to DB110. Data in this DB will be sent. The last input is input COM_RST, it's BOOL type and used to restart communication connection (interrupts the actual connection and creates a new connection). Each input are configured under the terms, which are defined in FC_SEND. Important parameter during setting-up of the inputs is REQ 
frequency, which defines how the data will be sent. In our case we set frequency to $2 \mathrm{~Hz}$, which is optimal for tracking states of PLC's inputs. (Figure 10). [5]

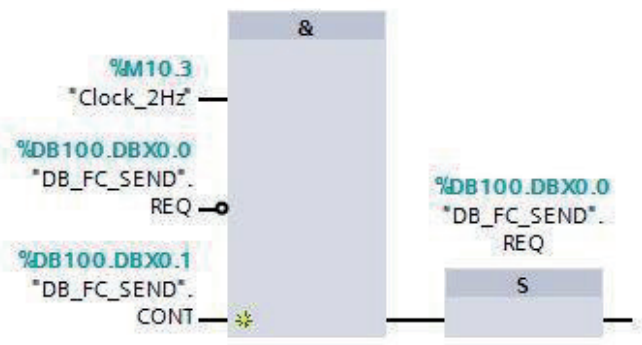

Fig. 10: Configuration of input REQ in TSEND_C block.

The second important parameter during the configuration of inputs is LEN, the maximal count of bytes. In our case its value is 10 (Figure 11).

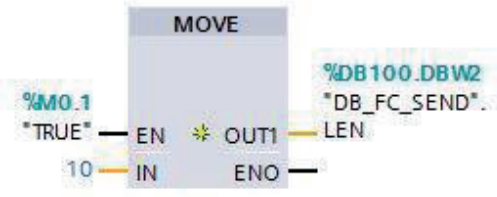

Fig. 11: Configuration of input LEN in TSEND_C block.

Outputs of TSEND_C are registered in DB100 too. The first output is DONE, of BOOL type. Value 0 means, that the sending hasn't started yet or is still in progress. Value 1 for variable DONE means, that the sending has been finished without errors. The second output is BUSY. BUSY is BOOL type, where value 0 means that the sending hasn't started yet, or has finished. Value 1 means that the sending is currently underway, and it's not possible to start new process of sending. Third output is ERROR. ERROR is type of BOOL. 0 means, that there were no errors during sending process. 1 means that an error occurred during sending. The last output is STATUS. It's type WORD and gives information about state of process.

FUNCTION for data receiving is FC_RECV. It's based on function block (FB) called TRCV_C (Figure 12). Similarly as FB for data sending, every input and output of this function block are registered in data block DB200. [5].

First input of this block is EN_R. It's type is BOOL and for value 1 it allows to receive data. This input is controlled by memory variable M0.1. Every other inputs are similar to inputs of TSEND_C block and

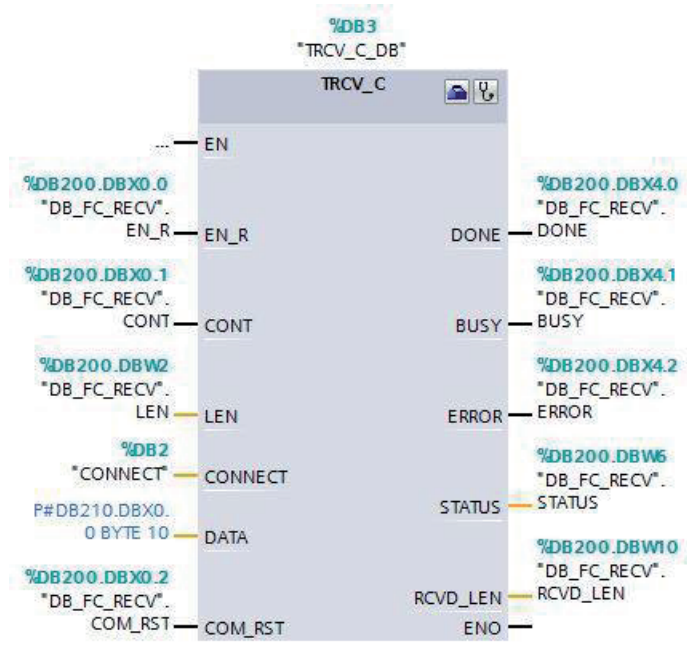

Fig. 12: Function block TRCV_C with inputs and outputs.

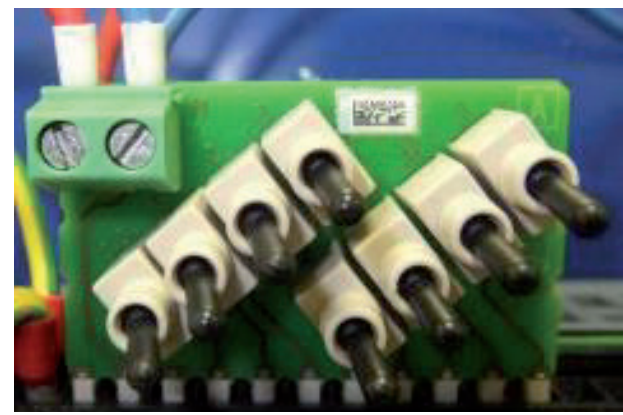

Fig. 13: Simulation module

has the same functions. [5]

Similarly the first four outputs of TRCV_C block are similar to outputs of TSEND_C block. There is a fifth output, RCVD_LEN. It's type of UINT and gives information about amount of currently received data in bytes. [5]

For easier manipulation and clearer preview, we chose to not rewrite input data for sending via VAT table, but we connected a simulation module on the first eight inputs of PLC (Figure 13). By switches on simulation module we can simulate presence of signal on the inputs of PLC: We will send this input byte.

For tracking received data we chose to not use VAT table, but we created program for HMI panel, where we can track signals of inputs on both PLC and by this method we can verify our connection.

\section{Program for HMI}

Creation of program for $\mathrm{HMl}$ panel is realized via TIA Portal. To visualize variables of PLC on HMI, 
it's needed to assign to PLC's variables tags. In TIA Portal there are two types of tags- PLC tags and $\mathrm{HMl}$ tags. During PLC configuration to PLC tags 10.0 to 10.7 of PLC1 we assigned PLC tags PLC1 10.0 to PLC1 10.7. Values of inputs of PLC2 we received via communication between PLC1 and PLC2 and by commands in OB1 block we registered them to memory on addresses M50.0 to M50.7. We assigned to these memory variables PLC tag PLC2 10.0 to PLC2 10.7. Tags for PLC2 were created in the same way. We created tag TRUE for memory bit M0.1, by which we can turn on or off the communication.

To created PLC tags we created HMI tags with same names. After connecting PLC tags to HMI tags, we can visualize our variables on HMI panels.

HMI program was created for panel KTP700 Basic PN. It's Root screen is shown on Figure 14.

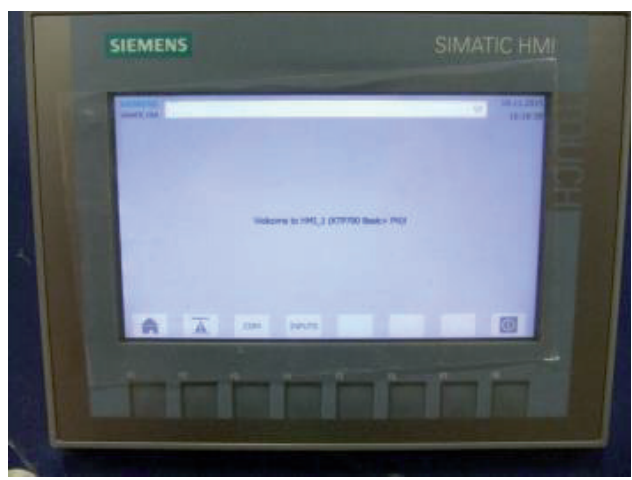

Fig. 14: Root screen for HMI.

To existing basic screens we created our screensCommunication and Inputs. We used template for every screen.

A switch is located on screen Communication (Figure 15), which allows to change value of memory variable M0.1 trough tag TRUE and therein turn on and off the communication. Also there is located signalization for ON state- green circle and for OFF state- red circle.

On screen Inputs (Figure 16) are located sixteen detecting lights. Eight for inputs on PLC1 and eight for inputs of PLC2. According to current state of inputs on each PLC the state of lights changes on this screen trough PLC and HMI tags, which were created and assigned to individual objects on the screen.

We can switch between screens either by functional hardware buttons on the body of $\mathrm{HMI}$ or by virtual buttons on the bottom of the touch screen.

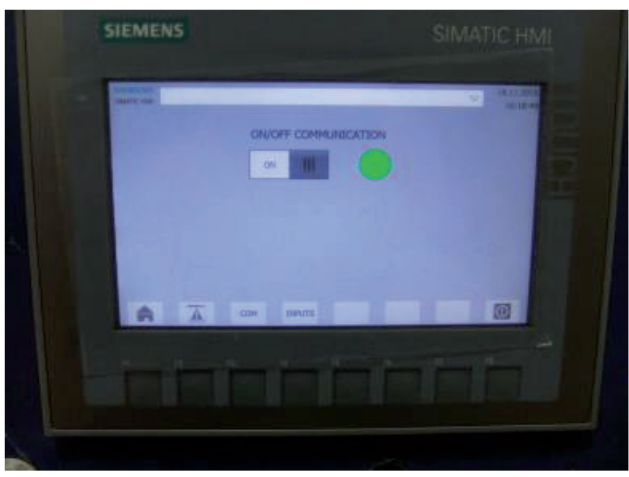

Fig. 15: Screen Communication.

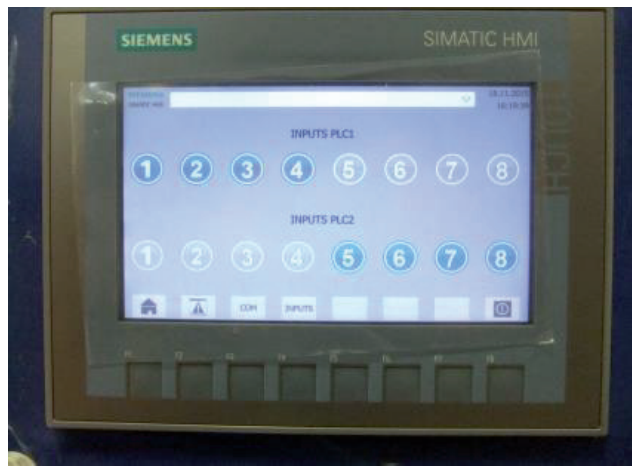

Fig. 16: Screen Inputs.

\section{Conclusions}

A model of the industrial network on PROFINET basis using the latest technology of SIEMENS was built for educational goals. The main components of that network were PLC Simatic S7-1200, HMI panel SIEMENS KTP700 Basic PN, switch SIEMENS SCALANCE XB005, and software products TIA Portal V13 SP1 version Basic, and Proneta.

Our first set of experiments with the model of the industrial network proved the functionality and obtained experience was start for generating practical tasks for students to solve them at practicals during courses for students in specialization automation.

Further plans will concentrate on enlarging the network with sensors and actuators, and development of practical tasks for testing of functionality of the network and measurement of its parameters. [6]

\section{Acknowledgments}

This work has been supported by the Slovak Grant Agency VEGA contract N. 1/0911/14 Implementation of wireless technologies into the design of new products and services to protect human health. 


\section{References and Notes}

[1] 6ES7214-1BG40-0XB0 Data sheet https://mall.industry.siemens.com/mall/en/WW/Catalog/Product/6ES7214-1BG40OXBO

[2] SIMATIC HMI Basic Panels 2nd Generation - Device overview. http://w3.siemens.com/mcms/human-machine-interface/en/operatorinterfaces/basicpanel/devices-secondgeneration/pages/default.aspx\#KTP900_20Basic

[3] 6GK5005-0BA00-1AB2 Data sheet. http://docseurope.electrocomponents.com/webdocs/1084/0900766b81084ae2. pdf

[4] SIMATIC STEP 7 Basic (TIA Portal). http://www.industry. siemens.com/topics/global/en/tia-portal/controller-sw-tiaportal/simatic-step7-basic-tia-portal/Pages/Default.aspx

[5] Open User Communication with TSEND_C and TRCV_C. http://support.automation.siemens.com/WW/view/ en/67196808

[6] Intenzifikácia výskumu a prepojenia na prax inováciou laboratórií / Dušan Šimšík, Alena Galajdová, Róbert Rákay - 2016. In: Automatizácia a riadenie v teórii a praxi: ARTEP 2016. Košice: TU, 2016 S. 52-1-52-10. - ISBN 978-80-553-2474-6

\section{Biographical notes}

Martin Višňovský, Ing. (1990), graduated at the Technical University of Košice in 2015 in field Automation. He is currently a PhD student at the Department of Automation, Control and Human Machine interaction at Technical University of Košice, Faculty of Mechanical Engineering, Main area of his research is Industrial automation.

Róbert Rákay, Ing. (1990), graduated at the Technical University of Košice in 2014 in field Automation. He is currently a PhD student at the Department of Automation, Control and Human Machine interaction at Technical University of Košice, Faculty of Mechanical Engineering, Main area of his research is Industrial automation.

Alena Galajdová, doc., Ing., PhD., (1961), graduated at the Faculty of Electrical Engineering at Technical University of Košice in 1984 in Radio electronics, associate professor in Automation since 2014, she is currently working as Deputy Head of Department of Automation, Control and Human Machine interactions (KARaKR) that belongs to Institute of Automation, Mechatronics and Robotics at Faculty of Mechanical Engineering Technical University of Košice. Her research is focused on Automation, Robotics, Modelling and Simulation and also in Biomedical Engineering, Rehabilitation Engineering and Neural Networks. She is author and co-author of more than 150 papers published in home and international journals and proceedings from conferences.

Dušan Šimšík, prof., Ing., PhD., (1950), full professor in Automation and control since 1997, currently working as head of the Department of Automation, Control and Human Machine interactions (KARaKR) that belongs to Institute of Atomation, Machatronics and Robotics at Faculty of Mechanical Engineering, Technical University of Košice. Graduated at the Faculty of Mechanical Engineering Technical University of Košice (1974), associate professor (1984), professor (1997). His research profile consists of Automation, Robotics, Automated
Manufacturing Systems, Modelling and Simulation and in period 1985-2011 also in Biomedical Engineering, Rehabilitation Engineering, and Assistive Technology for Disabled, Gerontechnology. He has been leading person for several domestic and foreign researchprojects (FP6 - MonAMI, DFA; FP7 - SMILING, eACCESS+, ETNA; national grant agencies VEGA, KEGA, MVTS, international educational projects TEMPUS, Socrates Grundtvig, Leonardo da Vinci). Acting as forensic expert in Machinery (since 1998). Author of several monographies and textbooks, numerous scientific and technical studies and articles.

He is a member of European Society of Biomechanics (ESB), International Society of Gerontechnology (ISG) and several national professional societies. He is a national contact and former member of the Board of Association for the Advancement of Assistive Technology in Europe (AAATE) and European Design for All e-Accessibility Network (EDeAN), chairman of ICTA Europe Rehabilitation International and IMEKO TC17. He is author and co-author of 5 books, 4 handbooks for students, over 200 papers published in home and international journals and proceedings from conferences. 